\title{
Dangerous liaisons: molecular basis for a syndemic relationship between Kaposi's sarcoma and $P$. falciparum malaria
}

\section{Katelyn L. Conant and Johnan A. R. Kaleeba*}

Department of Microbiology and Immunology, Uniformed Services University of the Health Sciences, Bethesda, MD, USA

\section{Edited by:}

Abraham L. Brass, University of Massachusetts Medical School, USA

Reviewed by:

Sam Mbulaiteye, National Cancer Institute, USA

Ann Moormann, University of

Massachusetts Medical School, USA

\section{*Correspondence:}

Johnan A. R. Kaleeba, Department of Microbiology and Immunology, Uniformed Services University of the Health Sciences, 4301 Jones Bridge Road, Bethesda, MD 20814, USA. e-mail: johnan.kaleeba@usuhs.edu
The most severe manifestations of malaria (caused by Plasmodium falciparum) occur as a direct result of parasitemia following invasion of erythrocytes by post-liver blood-stage merozoites, and during subsequent cyto-adherence of infected erythrocytes to the vascular endothelium. However, the disproportionate epidemiologic clustering of severe malaria with aggressive forms of endemic diseases such as Kaposi's sarcoma (KS), a neoplasm that is etiologically linked to infection with KS-associated herpesvirus (KSHV), underscores the significance of previously unexplored co-pathogenetic interactions that have the potential to modify the overall disease burden in co-infected individuals. Based on recent studies of the mechanisms that $P$. falciparum and KSHV have evolved to interact with their mutual human host, several new perspectives are emerging that highlight a surprising convergence of biological themes potentially underlying their associated co-morbidities. Against this background, ongoing studies are rapidly constructing a fascinating new paradigm in which the major host receptors that control parasite invasion (Basigin/CD147) and cyto-adherence (CD36) are, surprisingly, also important targets for exploitation by KSHV. In this article, we consider the major pathobiological implications of the co-option of Basigin/CD147 and CD36 signaling pathways by both P. falciparum and $\mathrm{KSHV}$, not only as essential host factors for parasite persistence but also as important mediators of the pro-angiogenic phenotype within the virus-infected endothelial microenvironment. Consequently, the triangulation of interactions between $P$. falciparum, KSHV, and their mutual human host articulates a syndemic relationship that points to a conceptual framework for prevalence of aggressive forms of KS in malaria-endemic areas, with implications for the possibility of dual-use therapies against these debilitating infections in resource-limited parts of the world.

Keywords: Kaposi's sarcoma, HHV-8, malaria, PfEMP-1, CD36, Basigin/CD147, PfRh5

\section{INTRODUCTION}

Plasmodium falciparum ( $P f$ ) malaria is one of the world's leading health challenges, and at least two million people, mainly children below the age of 5 years, die each year from clinical complications of the disease (Snow et al., 2005). The most severe manifestations of $P f$ malaria occur as a direct result of parasitemia following invasion of erythrocytes by post-liver blood-stage merozoites, and during subsequent cyto-adherence of parasitized red blood cells (pRBCs) to the vascular endothelium and other host cells and tissues. Erythrocyte invasion is executed by a family of $P f$ reticulocyte-binding-like homolog $(P f \mathrm{Rh})$ ligands displayed on the surface of merozoites. Among at least five known members of this family, PfRh5 (Rodriguez et al., 2008; Lopaticki et al., 2011; Tham etal., 2012), which is indispensable for merozoite growth in cultures, is essential for invasion by all $P f$ strains (Baum etal., 2009; Lopaticki etal., 2011). The cognate erythrocyte receptor for PfRh5 was recently identified as CD147 [also known as Basigin (BSG), extracellular matrix metalloproteinase inducer (EMMPRIN), and leukocyte activation antigen, M6 (hereafter CD147); Crosnier et al., 2011]. This discovery is notable for the fact that the CD147/PfRh5 pair is essential for invasion of all laboratory-adapted and field strains of $P f$, a crossstrain dependency that reveals opportunities for new anti-malarial therapies based on targeting this receptor/ligand interaction. Following invasion, extensive replication within infected human red blood cells (RBCs) results in surface expression of the multidomain $P f$ erythrocyte membrane protein-1 (PfEMP-1) family of genes (Baruch et al., 1995), including PfEMP-1 that serves as a platform for sequestration of pRBC from the blood circulation by adhering to endothelial and other host cells and tissues (Berendt, 1993).

The cyto-adhesive property of infected red blood cells to the microvasculature and sequestration within vital organ systems is an important survival strategy that allows the parasite to escape immune-mediated destruction (Urban et al., 1999). Cyto-adherence is mediated by an orchestrated set of interactions between specific regions within the ectodomain of PfEMP-1 [notable among them being the cysteine-rich interdomain region (CIDR1 $\alpha)$, with a variety of host molecules on the surface of capillary endothelial cells. A well-characterized host receptor that mediates cyto-adherence of most $P f$ isolates to the peripheral vasculature is human CD36 (Ockenhouse et al., 1989), although other 
cell adhesion molecules are also involved in execution of strain and tissue-specific cyto-adhesive events (Ockenhouse et al., 1991; Baruch et al., 1997; Figure 1).

Recent studies have revealed that while erythrocyte invasion and cyto-adherence represent essential evolutionary strategies for parasite growth, survival, and persistence, they are also invariably associated with alteration of cellular physiology, which in turn may contribute directly to the defining clinical manifestations of $P f$ infection (Trossaert et al., 1991; Fried and Duffy, 1998). However, less examined is the provocative hypothesis that malarial disease may not be solely attributable to complications associated with the various stages of the Pflifecycle alone; rather, the sequelae of illnesses associated with $P f$ malaria is likely to be the collective manifestation of a multitude of complex interactions between $P f$, other co-pathogenic infections, and the human host. In this article, we highlight emerging evidence supporting the proposition that the signaling pathways anchored by Basigin/CD147 and CD36, two of the known host receptors that control Pf invasion and cyto-adherence, respectively, are also targets for functional subversion by Kaposi's sarcoma (KS)-associated herpesvirus (KSHV), an inherently persistent cancer-associated herpesvirus that is prevalent in malaria-endemic regions. We discuss a number of surprising nodes of pathogenetic

\section{A Merozoite invasion and replication in red blood cells}
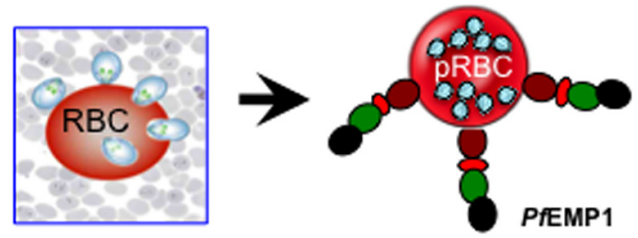

B Cytoadherence \& sequestration

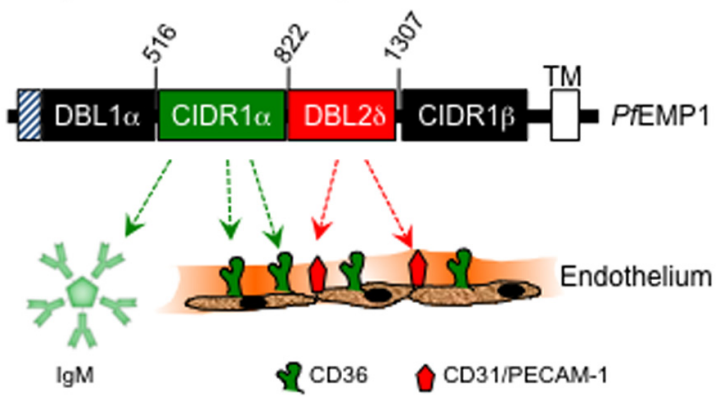

FIGURE 1 | Basic interactions of $\boldsymbol{P}$ falciparum with human erythrocytes during invasion $(A)$ and with blood capillary endothelium during

cyto-adherence (B). Upon exit from the liver, invasion of red blood cells by the blood-stage merozoites leads to replication and subsequent surface expression of the multi-domain PfEMP-1 molecule that mediates cyto-adherence via the binding activities of DBL1, CIDR1, and DBL2 domains of PfEMP-1 with various host adhesion receptors. Shown here is a region of PfEMP-1 encoded by FCR3S1.2-var1 (Chen et al., 2000). The CIDR1 domain primarily binds to CD36 and to members of the immunoglobulin superfamily, including IgM and CD31/PECAM (platelet endothelial cell adhesion molecule), whereas the DBL2 domain binds mainly to CD31/PECAM-1. interface between $P f$ and KSHV in this context, and evaluate the major implications of the apparent co-option, by both $P f$ and KSHV, of CD147 and CD36 signaling pathways as a means to promote $P f$ persistence on one hand, and virus-induced regulation of the angiogenic phenotype, on the other. We then provide a synthesis of how the triangulation of interactions between $P f, \mathrm{KSHV}$, and their mutual human host represents the basis for a venerable syndemic relationship that may explain the co-incidence of aggressive forms of $\mathrm{KS}$ in malaria-endemic regions.

\section{THE MULTI-FACTORIAL ETIOLOGY OF KAPOSI'S SARCOMA}

The most important pathological manifestation of KSHV infection is KS, a multifocal and highly angiogenic mesenchymal neoplasm characterized by profound inflammation and angioproliferative expansion of spindle cells believed to be of endothelial origin (Bubman and Cesarman, 2003). KS occurs in at least four epidemiological forms, each with its own distinguishing clinical disposition determined by age, sex, geographical location, socio-economic status, previous exposure to parasitic infections, co-infection with the human immunodeficiency virus (HIV), and the extent of acquired or iatrogenic immunosuppression (Bubman and Cesarman, 2003; Dourmishev et al., 2003; Haverkos, 2008): thus, iatrogenic KS is mostly associated with organ (especially renal) transplantation and is mostly seen as localized skin lesions among people from areas where KSHV is endemic. Epidemic HIV/AIDS-associated KS is more commonly seen among HIVinfected individuals, while classical KS (cKS) manifests among older men of Mediterranean origin as red to purple skin plaques or nodules primarily on the lower extremities. Endemic KS (eKS), which is strikingly similar to cKS in its clinical disposition, is highly prevalent in East and Central Africa, where it affects children and young adults as a cutaneous disease invading soft tissue and bone, or as a fulminant lymphadenopathy that can rapidly disseminate to visceral organs (Hengge et al., 2002a,b). eKS is currently the most common cancer in adult East and Central African men and follows only cervical and breast cancer in adult women (Bassett et al., 1995; Wabinga et al., 2000; Casper, 2006). An important distinction is that whereas iatrogenic and AIDS-related KS are invariably associated with an immunosuppressed state, cKS and eKS are generally not (Kestens et al., 1985), implying that the development and/or propagation of the latter two types of KS (i.e., cKS and eKS) may be controlled by unique, geographically restricted cofactors unrelated to HIV or drug-induced immune suppression (Pyakurel et al., 2007).

A number of non-competing hypotheses have been proposed to explain the contribution of socio-economic, behavioral, and environmental co-factors to the histogenesis of eKS and cKS, the two types of the lesion not strictly dependent on HIV or iatrogenic immunosuppression. For example, clinical studies have revealed that eKS displays a notable predilection for the feet and legs of rural peasants and cultivators living in highland areas, thus inspiring the volcanic soil hypothesis first proposed by Ziegler (1993) and subsequently supported by additional epidemiologic studies (Montesu et al., 1995; Montella et al., 1996, 1997, 2000; Goedert et al., 2002). This hypothesis proposes that walking barefoot allows soil-borne aluminosilicates, iron oxides, and other clay minerals to be taken 
up through sweat glands and abrasions by resident macrophages, dermal microvascular endothelial cells, and by the lymphatic system. The resulting chronic lymphatic irritation, inflammation, and immune suppression could in turn support primary infection through these portals, and/or reactivation of latent KSHV within the epidermal aspects of skin. Lin and colleagues also recently suggested that exposure to soil and water constituted an additional risk factor for KS development by promoting parasitic infections that could either reduce local immune reactivity or induce a condition of inflammation necessary for KS development as well (Lin et al., 2008). Alternatively, the finding that some natural products of plants indigenous to the areas with eKS could reactivate KSHV (Whitby et al., 2007) inspired the "oncoweed" hypothesis of KS development. Subsequently, Ruocco et al. (2011) noted that quinine, an anti-malarial drug used extensively as one of the mainstays for treatment of malaria in immunocompetent children, may trigger KS development via its immunosuppressive effects that could support virus reactivation. It is therefore noteworthy that although available evidence is not sufficient to explain the distinctive geographical distribution of $\mathrm{KS}$, the four known types of the lesion have a multi-factorial etiology, with the unifying theme being that the potential co-factors in each case operate at the level of the molecular mechanisms that control KSHV infection, dissemination, and the balance between virus replication and establishment of latency. This is a relevant link given the fact that a sustained state of low level lytic replication may be important for histogenesis and probably propagation of the KS lesion (Grundhoff and Ganem, 2004).

\section{CO-PATHOGENIC MECHANISMS OF KS AND MALARIA: A HYPOTHESIS}

Like Burkitt's lymphoma, KS is one of the most prevalent childhood cancers in malaria-endemic areas. The incidence of KS displays a considerable degree of geographic variation that mirrors the prevalence of its causative agent, and may depend on etiologic mechanisms that are controlled by geographically restricted cofactors, including malaria endemicity (Ziegler et al., 1984, 2003; Ascoli et al., 2001, 2006a,b; Coluzzi et al., 2003, 2004b; Wakeham et al., 2011). For example, KS incidence is particularly high in sub-Saharan Africa, a region with one of the highest rates of malaria deaths (Snow et al., 2005). Epidemiological studies have also shown a disproportionately high incidence of KS among elderly men in Greece, Turkey, Israel, and in Italy where the greatest number of recorded cases are in the formerly malaria-endemic provinces of Sardinia, Sicily (Vitale et al., 2001), and in the Po valley (Ascoli et al., 2001). Such overlapping geographic clustering of $P f$ malaria with eKS or cKS has inspired the co-pathogenesis hypothesis, which proposes that a previous or ongoing exposure to KSHV in a setting of underlying parasite persistence (or vice versa) may result in molecular interactions between $P f$ and KSHV that could modify the overall disease burden exerted by both pathogens at a micro level. Based on this attribution, we refer to the putative co-morbidity of KS and Pf malaria (in settings for which the evidence for this linkage is strong), as representing an example of a classic syndemic relationship articulated by the display of co-incident clustering within defined geographic regions.
In spite of evidence from case-control studies that show a disproportionately high KS prevalence in areas of currently or previously high malaria endemicity, a molecular link between KS pathogenesis and $P f$ malaria has not been rigorously examined at a micro level, and the correlation is even more difficult to establish at a population level because of the indeterminate nature of the mechanisms by which malaria might influence KS pathogenesis outside the known clusters of endemic disease co-incidence. On one hand, the role of malaria as a co-factor for eKS has been hypothesized based on the potential of the Anopheles mosquito vector to contribute to person-to-person spread of $\mathrm{KSHV}$, and to establish an immunosuppressed state at the site of the mosquito bite, which would then create a local permissive environment for KSHV infection (Coluzzi et al., 2003; Ascoli et al., 2006a,b,c). However, we propose an alternative model based on the provocative hypothesis that both the malaria parasite and KSHV exert bidirectional influences upon each other that operate at a much more complex level beyond the permissive benefits of immune suppression or the modifying effects of occupational, socio-economic, or environmental co-factors.

As a conceptual basis for our hypothesis, consideration of some of the known molecular controls that regulate the KSHV life cycle reveals a number of insights into the potential pathobiological link between KS and malaria. Like Epstein-Barr virus (EBV) and related herpesviruses, KSHV establishes both lytic and long-term latent infections, the balance between which determines the timing and intensity of pathologic outcomes in specific organ systems. Interestingly, both KSHV and EBV are highly prevalent in malariaendemic areas and also share many features in their life cycles, including the manner in which they regulate the molecular switch between latency and lytic replication. For example, expression of the major KSHV replication and transcription activator (RTA) is essential for virus replication and dissemination, and is dependent on activation of p38 and extracellular signal-related kinase 1/2 (ERK1/2) mitogen-activated protein kinase (MAPK; Xie et al., 2008). KSHV RTA is the genetic and functional homolog of EBV RTA/BRLF1 (Bam HI fragment Z rightward open-reading frame 1) which, in concert with Zebra Transcriptional Activator/BamHI fragment $Z$ rightward open-reading frame 1 (ZTA/BZLF1), can initiate EBV lytic cycle (Staudt and Dittmer, 2007). KSHV RTA positively regulates immediate and delayed early promoters as well as its own promoter by interacting with cellular transcription factors such as activator protein-1 (AP-1), octamer binding transcription factor 1 (Oct-1), recombination signal-binding protein Jkappa (RBP-Jк), and CCAAT/enhancer binding protein alpha (C/EBP $\alpha$; Staudt and Dittmer, 2007); in this way, RTA is susceptible to a variety of signal transduction pathways that are likely to activate its promoter. In fact, KSHV RTA expression (and therefore lytic replication) can be induced in vitro using a variety of chemical compounds such as ionomycin (a calcium ionophore), sodium $n$ butyrate $(\mathrm{NaB}$, a histone deacetylase inhibitor) and phorbol esters such as 12-O-tetradecanoylphorbol-13-acetate (TPA), all of which can also be used to induce EBV lytic cycle (Luka et al., 1979; Renne et al., 1996; Zhu et al., 1999; Gao et al., 2001).

Given the shared genetic and biological properties of EBV and $\mathrm{KSHV}$, it is not surprising that the two viruses can co-exist as latent episomes in certain peripheral effusion lymphoma-derived 
cell lines (Horenstein et al., 1997). They also display similarities not only in the mechanism of induction of the lytic cycle but also in the distribution of endemic cancers associated with them in regions of high malaria endemicity, which perhaps reflects the contribution of malaria as a common co-factor in the pathogenesis of cancers associated with these viruses. For EBV, the epidemiological association between malaria and African endemic Burkitt's lymphoma (eBL) is well established (Moormann et al., 2011), although the molecular mechanisms by which malaria modifies eBL and other pathobiological outcomes of EBV infection remain a matter of intense investigation (Rochford et al., 2005). Interestingly, eBL is a B cell lymphoma propagated by a deregulation in the $c-m y c$ oncogene as a result of chromosomal translocation, and although EBV is a necessary etiological agent for eBL, it is clearly not sufficient in absence of other essential co-factors. It is also worth noting that whereas nearly all African children in endemic areas suffer from several malaria episodes as a result of chronic exposure to $P f$, only a fraction of them show signs of severe, life-threatening forms of the disease, implying that the biological processes underlying the progression of infection to disease are much more complex. Interestingly, EBV is ubiquitous in the general human population, and in children living in malaria endemic areas, primary infection can occur within a few months of birth (Piriou et al., 2012), and may seroconvert within 3 years after primary infection, followed by a tightly orchestrated viral latency state in memory B cells that reflects the balance between viral replication and host immune control. Emerging new evidence now suggests that the exit from latency to the viraemic state that supports eBL development may be impacted at a molecular level by the replicative blood-stage form of the parasite life cycle. Thus, Chene et al. (2007) recently demonstrated that interactions between the CIDR $1 \alpha$ domain of PfEMP-1 on the surface of pRBCs, with a cognate surface receptor(s) expressed on the surface of EBV-infected human memory B cells, stimulates B cell proliferation (as previously shown; Donati et al., 2004) and reactivates EBV not only from the chronically infected Akata cell line but also from latently infected B cells isolated from the peripheral blood and tonsils of healthy EBV carriers (Chene et al., 2007). The mechanism by which conjugation of PfEMP-1-expressing pRBC with EBV-infected B cells triggers virus replication in this model remains to be elucidated, but the consequences of this interaction can be readily appreciated as providing a possible explanation for the increased EBV viral load that constitutes risk for eBL among children living in areas of high malaria endemicity.

Remarkably, we have also discovered that cross-linking of CD36 on the surface of KSHV-infected cells with MC179, a recombinant peptide derived from the CIDR $1 \alpha$ domain of PfEMP-1 that normally interacts with CD36 to mediate cyto-adherence (Ockenhouse etal., 1989, 1991; Baruch et al., 1997), not only upregulated CD36 expression (Figure 2A) but also reactivated the virus from latency through transcriptional activation of KSHV RTA (Figure 2B), and that the molecular mechanisms that control this process overlap with those that putatively regulate PfEMP-1dependent EBV reactivation from latently infected cells (Chene et al., 2007). Remarkably, structural mimics of MC179, including the two helical heptad repeats (HR1 and HR2) derived from KSHV entry glycoprotein B (gB) also upregulated CD36
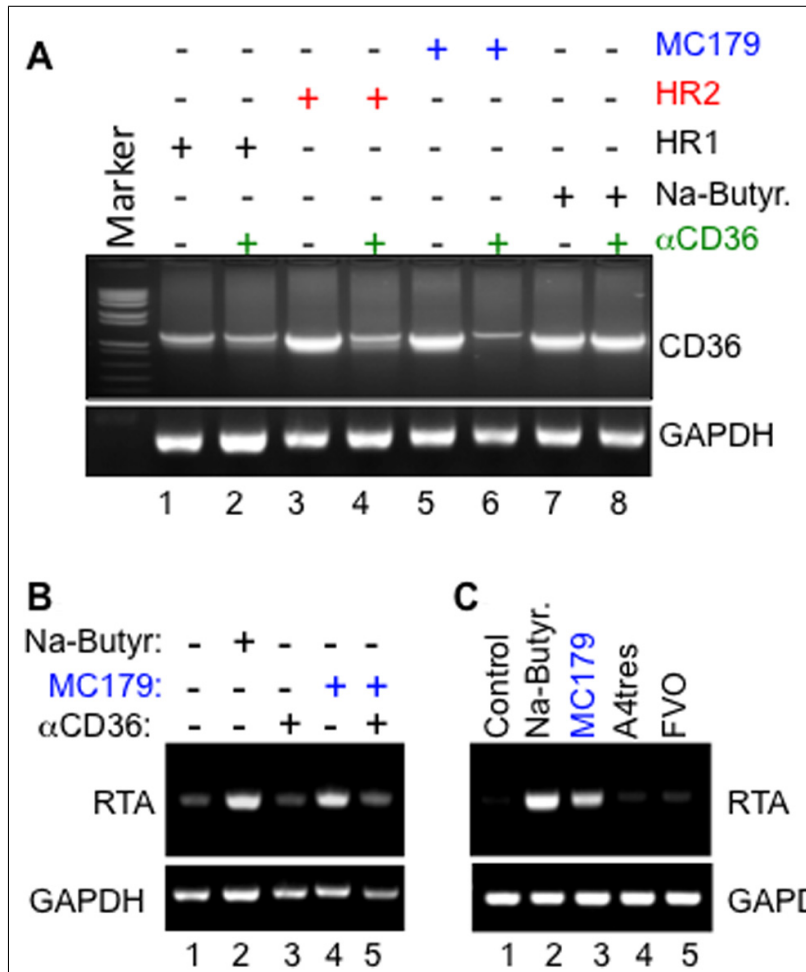

C

FIGURE 2 | (A) Upregulation of CD36 by both the PfEMP-1-CIDR1 $\alpha$-derived MC179 peptide (lanes 5 and 6 ) and by KSHV gB-derived heptad peptide ligands HR2 (lanes 3 and 4), and the less potent HR1 (lanes 1 and 2) are both blocked by a monoclonal anti-CD36 antibody. Note that anti-CD36 did not block control upregulation of CD36 in response to treatment with sodium butyrate (NaB; lanes 7 and 8). (B) Activation of KSHV RTA by MC179 occurs via a CD36-dependent mechanism that can be blocked by anti-CD36 antibody FA6-152. Methodology: briefly, melanoma-derived Mel1700 cells were seeded in a six-well plate and either left untreated or pre-incubated with $10 \mu \mathrm{g} / \mathrm{ml}$ of anti-CD36 monoclonal antibody clone FA6-152 for $25 \mathrm{~min}$. at $25^{\circ} \mathrm{C}$. After washing to remove excess antibody, cells were incubated with either $100 \mu \mathrm{g} / \mathrm{ml}$ of KSHV gB-derived HR1, $200 \mu \mathrm{g} / \mathrm{ml} \mathrm{HR} 2$ peptide, $100 \mu \mathrm{g} / \mathrm{ml}$ recombinant MC179, or $2 \mathrm{mM}$ sodium butyrate $(\mathrm{NaB})$. Forty-eight hours after treatment, total RNA was isolated and used as template in semi-quantitative RT-PCR with primers to an internal fragment of human CD36, viral RTA, or human glyceraldehyde 3-phosphate dehydrogenase (GAPDH) loading control. (C) Specific activation of the KSHV lytic switch protein, RTA, by the PfEMP-1-CIDR1 $\alpha$ derived peptide MC179 from Pf Malayan Camp strain, but not by peptides derived from CIDR $1 \alpha$ domains of A4tres (which binds ICAM-1) or the Vietnam Oak Knoll strain (FVO).

expression and further induced virus reactivation in a CD36dependent manner. Indeed, like MC179, the effect of these helical peptides could be blocked by a monoclonal antibody to CD36 (Figures 2A,B), suggesting that they bind a region on the exoplasmic face of CD36 that overlaps with MC179. It is also insightful that the KSHV lytic cycle was activated only by the PfEMP-1CIDR $1 \alpha$-derived peptide from Pf Malayan Camp strain, but not by peptides derived from the Vietnam Oak Knoll (FVO) or A4tres strain [that preferentially binds intercellular adhesion molecule 1 (ICAM-1) and not CD36] (Figure 2C), demonstrating that reactivation of KSHV by the parasite ligand displays some degree of strain specificity for $P f$ that is associated with severe malaria in Africa. 
Since CD36 mediates parasite persistence by mediating cytoadherence and sequestration of parasitized erythrocytes away from immune surveillance, the ability of MC179 to stimulate $\mathrm{KSHV}$ reactivation is significant, as it supports a model whereby in vivo cross-linking of CD36 on the surface of KSHV-infected cells, either by its natural ligand(s) or upon conjugation with the CIDR $1 \alpha$ domain of PfEMP-1 expressed on the surface of parasitized erythrocytes (as illustrated in Figure 3), represents a previously unrecognized mechanism by which KSHV lytic replication could be induced in the context of a $P f$ malaria co-infection. This model provides many new opportunities for experimental examination of the ability of parasite-derived antigens to reactivate KSHV during cyto-adherence on the surface of infected blood endothelial cells and within tissues and organs: (i) Biochemical analysis of structure/function relationships that control interactions of CIDR1 $\alpha$ (and its structural analogs) with CD36, should reveal potential targets for small-molecule inhibition of parasiteinduced sequestration or reactivation of KSHV in co-infected individuals. (ii) Elucidation of the signaling mechanisms that regulate CD36-dependent RTA activation will require multi-pronged approaches that employ dominant negative versions of the Srclike kinases such as yes, fyn, and lyn that control down-stream signaling events initiated by CD36 ligation (see Figure 3). (iii) Deletional mutagenesis, domain-swapping, functional complementation, and loss-of-function analysis of genetic mimics of the recently identified CD36 polymorphisms that lack the signaling motif (Aitman et al., 2000; Gelhaus et al., 2001; Chilongola et al., 2009; Fry et al., 2009) should elucidate the correlates of upstream signaling networks required for CD36-dependent activation of the KSHV lytic cycle.

\section{CD36 AND THE Pf LIFECYCLE: IMPLICATIONS FOR KSHV PATHOGENESIS IN THE CO-INFECTED HOST}

CD36 is a class II glycoprotein involved in multiple physiological functions including cell adhesion, fatty acid uptake, non-opsonic phagocytosis, and angiogenesis (McGilvray et al., 2000; Podrez et al., 2007; Silverstein and Febbraio, 2007, 2009). The CD36 structure consists of a large extracellular loop and two short cytoplasmic tails at the $\mathrm{N}$ - and C-termini (Silverstein and Febbraio, 2009). The C-terminal tail is involved in signal transduction via association with $S r c$-like kinases, whereas the extracellular domain contains binding sites for thrombospondin-1 (TSP-1), a potent natural inhibitor of angiogenesis (Bagavandoss and Wilks, 1990; Good et al., 1990; Tolsma et al., 1993), and a variety of other ligands including the CIDR1 $\alpha$ domain of PfEMP-1 (Ockenhouse et al., 1989). Although CD36 has never been directly associated with KSHV pathogenesis, some of its pleiotropic functions are consistent with its potential contribution to the basic pathobiology of KSHV. For example, CD36 is often expressed in association with signaling structures such as lipid rafts that also contain host receptors for KSHV entry, including integrins and the KSHV fusion receptor complex xCT/CD98 (Akula et al., 2002; Kaleeba and Berger, 2006; Veettil et al., 2006). Since CD36-mediated signaling may culminate in activation of p38 and ERK MAPK pathways that overlap with one or more pathways necessary for KSHV RTA-dependent transcription of viral gene expression (Yipp et al., 2003; Cohen et al., 2006; Xie et al., 2008), our data suggests that

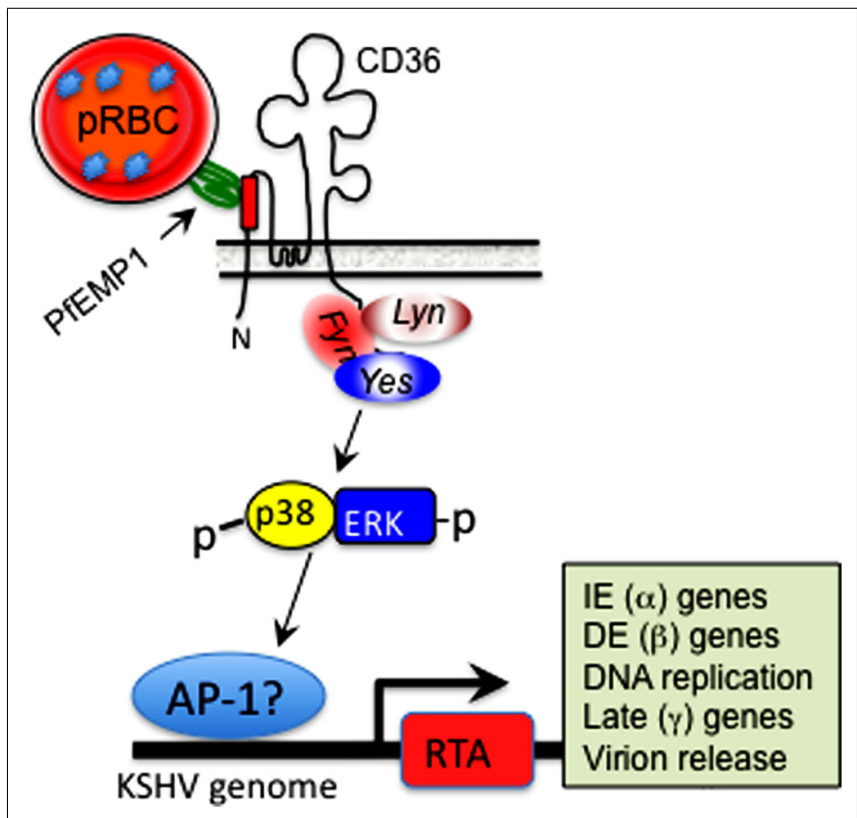

FIGURE 3 | Hypothetical model of CD36-dependent RTA activation. A motif displayed by PfEMP-1 on the surface of parasitized erythrocytes interacts with its cognate epitope within the ectodomain of CD36 on the surface of microvascular endothelial cells. This interaction activates one or more of the Src-like kinases, which in turn initiate a phosphorylation cascade that results in $\mathrm{p} 38$ and ERK/MAPK activation. This process culminates in activation of a cellular transcription factor, likely AP-1 (dimer of $c$-jun and $c$-fos), which translocates into the nucleus and stimulates KSHV RTA-dependent transcription of viral lytic cycle genes, starting with immediate early (IE), which then activate delayed early (DE), followed by late structural genes involved in assembly of an infectious virus particle.

CIDR1 $\alpha$-dependent RTA activation may occur via by signals transduced through CD36 (Figure 3). However, our model also raises many significant questions: (a) Does CIDR1 $\alpha$-induced KSHV replication involve mechanisms that overlap with, or distinct from those that support CIDR $1 \alpha$-induced EBV replication (Chene et al., 2007)? (b) Does it require the traditional CD36-regulated recruitment of Src-like kinases and subsequent phosphorylation of p38 and ERK MAPK? (c) Does it directly result in downstream activation of a specific cellular transcription factor such as AP-1 that is known to activate KSHV RTA (or EBV ZTA) promoter(s) (e.g., see Figure 3)? (d) Is it strictly dependent on interactions of CIDR $1 \alpha$ with a distinct epitope on CD36, or does it overlap with, and is therefore also inducible by, other CD36 ligands such as TSP1 ? Investigation of the role of TSP-1 in this context is relevant, given that TSP-1 is present in high concentrations in human saliva (Crombie et al., 1998, 2001; Shugars, 1999) that generally contains significant levels of KSHV virions shed from the underlying oral zones of carriers (Pauk et al., 2000; Coluzzi et al., 2004a; Chene et al., 2007; Hadinoto et al., 2009). Critical new experiments that address these derivative questions represent an exciting area of research into the molecular basis for the emerging new paradigm of co-pathogenesis. It is also anticipated that isolation of polymorphisms in CD36 and other human genes that control host interactions with $P f$, EBV and KSHV may open up additional opportunities for population-level studies aimed at explaining the 
overlapping distribution of KS, Burkitt's lymphoma, and malaria in areas where these diseases display coincident endemicity.

\section{VIRAL SUBVERSION OF THE CD36 SIGNALING PATHWAY: IMPLICATIONS FOR PARASITE SEOUESTRATION AND ANGIOGENESIS}

Angiogenesis, defined as the development of new blood vessels, is necessary for growth and proliferation of vascular tumors like $\mathrm{KS}$, the extent of which is controlled by the balance between pro-angiogenic and angiostatic elements of the human hemostatic system. One of the regulatory components of this system is TSP-1, the angiostatic CD36 ligand known to inhibit endothelial cell proliferation, migration, and tube formation (Iruela-Arispe et al., 1991). Remarkably, KSHV upregulates CD36 in melanomaderived cell lines but downregulates the protein in endothelial cells both at mRNA and protein levels (Figure 4). The mechanism(s) by which KSHV accomplishes these dichotomous, cell type-specific effects are not fully understood, but one study recently demonstrated that KSHV-encoded microRNAs can directly target TSP-1 mRNA for degradation (Samols et al., 2007), ostensibly to promote an angiogenic growth state of infected cells via attenuation of the angiostatic effects resulting from interactions between TSP1 and CD36. Since the angiostatic signal is associated with viral lytic replication while the angiogenic phenotype is linked to the latency phase, virus regulation of CD36 expression and signaling in infected cells implies that the correlates of KSHV latency are contextually linked to the angiogenic phenotype in diseaserelevant cells. They also provide a potential explanation for our recent findings that the KSHV latency program is inefficient in melanoma cells from which the virus undergoes robust spontaneous replication, as opposed to endothelial cells in which the virus establishes a much tighter state of latency (Fontana et al., unpublished findings).

In addition to direct targeting of TSP-1, KSHV blunts CD36 signaling in endothelial cells by upregulating the endothelin (ET-1) system. ET-1 is a pro-angiogenic peptide secreted by the vascular endothelium and its deregulation is implicated in the pathogenesis of many malignancies (Nelson et al., 2003). ET-1 polypeptides and their cognate receptors are expressed in KS lesions (Nelson et al., 2003; Basilico et al., 2004), and ET-1 receptor blockade limited $\mathrm{KS}$ cell invasion in an in vivo tumor growth model (Rosano et al., 2003). The interplay between the pro-angiogenic effects of ET1 and the pathophysiology of KS is also encountered in patients with complicated Pf malaria in which plasma concentrations of big ET-1, the precursor for bioactive ET-1, are elevated (Wenisch et al., 1996) as a direct result of cyto-adherence of pRBCs to human endothelial cells, independent of the parasite strain and regardless of the origin of endothelial cells (Basilico et al., 2004). There is, therefore, a significant degree of molecular crosstalk between Pf and KSHV at the level of ET-1 biology. Considered in a broader context, it is conceivable that by suppressing the angiostatic effects of CD36 signaling either by reducing CD36 expression, downregulation of TSP-1, or via upregulation of ET-1, KSHV could establish long-term persistence by establishing a state of limited CD36-dependent viral reactivation, or reduce CD36-dependent cyto-adherence and consequently limit the frequency of illnesses associated with this aspect of the parasite life cycle. Unfortunately, the latter outcome might be associated with an increase in the likelihood for parasite access to the extra-peripheral organs such as the brain, which may elevate the probability of cerebral malaria. Although measurement of these parameters in vivo is not trivial, a number of guiding principles can emerge from in vitro scrutiny of these molecular interactions based on experimental approaches that might predict their occurrence in vivo.

\section{CD36 POLYMORPHISMS DO NOT PROTECT FROM MALARIA: PERSPECTIVES ON SELECTION PRESSURE}

It was recently discovered that many people of African origin harbor a high frequency of mutations and single-nucleotide polymorphisms (SNPs) that cause a deficiency in the CD36 gene, yet

\section{A sqRT-PCR}

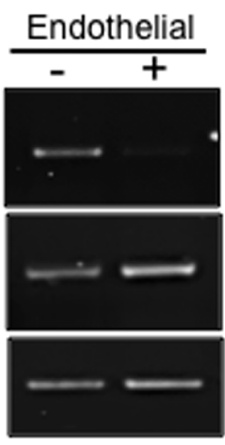

\section{B Western blot}

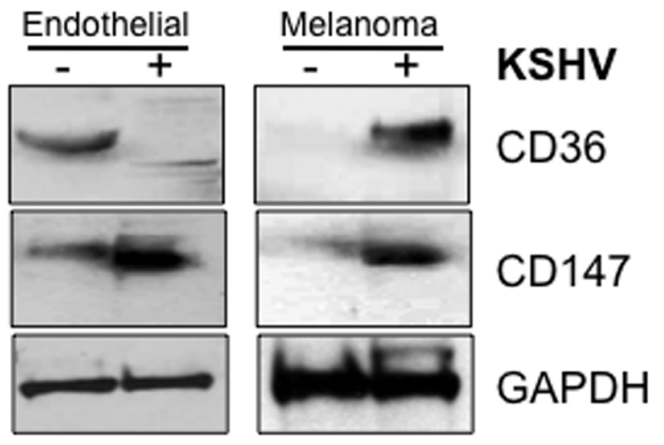

FIGURE 4 | Cell type-specific outcomes of KSHV infection in skin versus endothelial cells with respect to CD36 and CD147 expression.

Semi-quantitative RT-PCR (A) and Western blot (B) analysis of CD147 and

CD36 expression in uninfected (-) versus infected $(+)$ endothelial (lymphatic) or melanoma-derived Mel1700 cells. KSHV upregulates both CD36 and

CD147 in melanoma-derived cells, whereas in lymphatic and telomerase-immortalized mixed dermal microvascular and brain endothelial cells, KSHV upregulates CD147 (confirming a recent study; Qin et al., 2010) but downregulates expression of CD36 (and its angiostatic ligand, TSP-1; data not shown); in vivo, these dichotomous effects are consistent with promotion of angiogenesis, invasion, and tumor metastasis in disease-relevant cell types. 
they still suffer from severe (particularly cerebral) malaria (Aitman et al., 2000; Gelhaus et al., 2001; Chilongola et al., 2009; Fry et al., 2009). The SNPs found in Kenya and Gambia introduce a premature stop codon that results in a truncated CD36 protein lacking the C-terminus and is therefore incompetent for signal transduction but can still bind its ligand(s), leading to the conclusion that mutations that cause CD36 deficiency may reduce CD36-mediated parasite sequestration in peripheral organs but they may not protect from severe cerebral malaria (Aitman et al., 2000) that is associated with cyto-adherence to brain endothelium via interactions of PfEMP-1 with ICAM-1 but not CD36 (Ockenhouse et al., 1991). This level of linkage in which mutations in CD36 - a molecule that is important for parasite persistence cause a deficiency that does not alter malaria pathogenesis, implies existence of selection pressures that may be induced or maintained in the population by an endemic infection (other than malaria) whose persistence is linked to this genetic output.

The fact that the mutations and their phenotypes occur at high frequency in malaria-endemic areas with a high prevalence of viruses associated with endemic cancers underscores a pathobiological paradigm whereby an inherently persistent tumor virus such as KSHV (or EBV) could provide the selective pressure for introduction or maintenance of such a mutation into the genetic registry of populations living in regions of high malaria endemicity. Given that PfEMP-1 interactions with CD36 result in induction of the viral lytic cycle, such a virus-induced genetic output would conceivably be designed to promote virus escape from immune surveillance by limiting virus reactivation that might result from interactions between PfEMP-1 and CD36 on latently infected cells. The impact of such a genetic influence, can only be measured against the host's ability to restrict virus replication and dissemination, and it could be achieved by interrogating viral genomic variability or stability in a given population against a profile of polymorphisms within the genetic registries at the CD36 locus on a population basis. Availability of patient samples with known patho-status and disease severity from regions in which the distribution of malaria overlaps with the incidence of virus-associated endemic cancers would facilitate such a retrospective analysis.

\section{CD147 AND KSHV PATHOGENESIS}

CD147 is a widely expressed, type I integral membrane receptor that belongs to the immunoglobulin (Ig) superfamily (Biswas et al., 1995). It is over-expressed in a variety of disseminated human solid cancers and is a major contributor to the malignant phenotype in a variety of human cancers (Riethdorf et al., 2006). Signaling events transduced through CD147 are associated with survival, metastasis, and invasion of a variety of cancer cells, mainly because it stimulates enhanced stromal release of multiple matrix metalloproteinases (MMPs) and vascular endothelial growth factor (VEGF), which are among the key mediators of angiogenesis and metastatic transition (Marieb et al., 2004; Tang et al., 2005; Bougatef et al., 2009; Kanekura and Chen, 2010). CD147 promotes hyaluronan synthesis (Marieb et al., 2004; Slomiany et al., 2009a), upregulates the Wnt/ $\beta$-catenin signaling pathway (Sidhu et al., 2010), and is also involved in epithelial-to-mesenchymal transition (EMT; Wu et al., 2011). Given the pleiotropic function of CD147, the significance of CD147/PfRh5 interactions in parasite invasion unlocks new avenues for investigating the nodes of pathogenetic intersection between $P f$ malaria and other coinfecting agents such as KSHV that have evolved mechanisms to subvert the CD147 signaling pathway to promote their existential success.

Several layers of this pathogenetic intersection are revealed by new data on the manner in which both $P f$ and KSHV have evolved to exploit CD147 and endothelial cell biology:

a. Whereas endothelial cells play a prominent role as a platform for cyto-adherence of pRBCs, KSHV displays profound tropism for this cell lineage that represents the basis for origination of the hyper-proliferating spindle cells characteristically found in KS lesions (Bubman and Cesarman, 2003; Ganem, 2006).

b. Recent studies demonstrated compelling evidence that de novo $\mathrm{KSHV}$ infection of human endothelial cells as well as oral and fore-skin-derived fibroblasts results in upregulated expression of CD147 and that this effect is directly associated with various virological outcomes consistent with a pro-invasive, migratory, and pro-angiogenic phenotypes (Qin et al., 2010; Dai et al., 2012a,b). We have also found that KSHV upregulates CD147 not only in melanoma-derived cells but also in chronically infected lymphatic, microvascular, and brain endothelial cells (e.g., see Figure 4).

c. Two recent reports showed that KSHV promotes endothelialto-mesenchymal transition (endo-MT) through activation of Notch-dependent signaling events that culminate in stimulation of an invasive phenotype analogous to that orchestrated by CD147 (Cheng et al., 2011; Gasperini et al., 2012). KSHV-induced endo-MT was dependent on the activity of membrane-type-1 MMP (MT1-MMP; Cheng et al., 2011), which is consistent with a role for CD147 in endo-MT since MT1-MMP is a CD147-stimulated endopeptidase involved in extracellular matrix remodeling. It is therefore conceivable that one of the mechanisms underlying KSHV-induced endo-MT may involve viral induction of MMP activity (Qian et al., 2007), likely through upregulation of CD147.

d. CD147 has been implicated in the entry processes of a number enveloped viruses including HIV-1 (Pushkarsky et al., 2001), measles virus (Watanabe et al., 2010), and severe acute respiratory syndrome coronavirus (SARS-CoV; Chen et al., 2005). Based on our recent studies, we also have reason to believe that CD147 may regulate KSHV entry as well, as an antihuman CD147 (neurothelin) antibody can potently block KSHV glycoprotein-mediated fusion, consistent with existence of CD147 in the membrane of KSHV-permissive cells as part of a molecular supercomplex (Guo et al., 2000; Yang et al., 2007; Gallagher et al., 2009) that includes host molecules implicated in virus entry, such as integrins (Akula et al., 2002) and the cystine transporter complex xCT/CD98hc (Xu and Hemler, 2005; Kaleeba and Berger, 2006). It is also noteworthy that, like CD36, CD147 also associates with the xCT/CD98hc complex and confers resistance to some chemotherapeutic drugs (Okuno et al., 2003; Yang et al., 2007; Zou et al., 2007). Interestingly, another independent study also demonstrated that the transport activity of $\mathrm{xCT} / \mathrm{CD} 98 \mathrm{hc}$, perhaps in association with CD147, is a critical correlate of resistance to cisplatin (Huang et al., 2005; 
Singh et al., 2010; Chen etal., 2011; Riglar et al., 2011). For KSHV-infected individuals, one implication of these molecular associations could be that virus-induced upregulation of CD147 (as we and others have shown; Qin et al., 2010; Dai et al., 2012a,b), could stabilize CD147-containing multi-partite complexes and in turn potentiate their drug efflux functions, which could blunt the efficacy of chemotherapeutic strategies that might be used in the treatment of KS and other virusassociated malignancies. In support of this view, Qin et al. (2011) recently showed that the intrinsic resistance of KSHVpositive peripheral effusion lymphomas to the cytotoxic effects of paclitaxel and doxorubicin depends on orchestrated interactions of CD147 with lymphatic vessel endothelial receptor 1 (LYVE-1) and the homodimeric ATP-binding cassette (ABC)G2/BCRP (breast cancer resistance protein) drug transporter that is highly expressed on the surface of primary effusion lymphoma (PEL)-derived cell lines.

e. Available evidence suggests that "outside-in" signaling may be required for the malaria parasite invasion (Singh et al., 2010; Chen et al., 2011; Riglar et al., 2011), but it remains to be determined if the structural framework that supports erythrocyte invasion through CD147 is distinct from, or overlaps with, the epitope on the CD147 ectodomain that senses signals transduced to endothelial and other cells that express this molecule. If they are the same, PfRh5-mediated conjugation of merozoites with CD147 on normal or infected endothelial cell surfaces should result in so-called parasite-to-host "trans-signaling" events that may lead to untoward pathologic outcomes unrelated to parasite invasion itself. Given that PfRh5 has neither a transmembrane domain nor a cytoplasmic tail, the probability of trans-signaling is likely to be high since a soluble form of PfRh5 [existing either as a monomer, as part of a bioavailable complex with another pathogen molecule such as PfRipr (Chen et al., 2011), or as bound to a host "handler"] could initiate CD147-dependent signaling outcomes that are likely to modify Pf malaria or KSHV infection (Marieb et al., 2004; Xu etal., 2007; Ruiz et al., 2008; Slomiany et al., 2009b; Sidhu etal., 2010; Wu etal., 2011). A first-line experimental testing of such a concept should seek to determine whether blood-borne merozoites or soluble forms of PfRh5 can indeed bind CD147 on KSHV-infected cells and whether those interactions are directly associated with a CD147-dependent alteration in cellular behavior, including extracellular remodeling and induction of a pro-angiogenic phenotype that is one of the defining features of the infectious process of KSHV (Qin et al., 2010; Cheng et al., 2011; Dai et al., 2012a,b; Gasperini et al., 2012). In Figure 5, we highlight some of the important nodes of intersection between KSHV and the malaria parasite in endothelial cells and skin-derived melanoma cells, along with their potential impact on the virus life cycle (i.e., reactivation), KS tumorigenesis, and malaria disease outcomes.

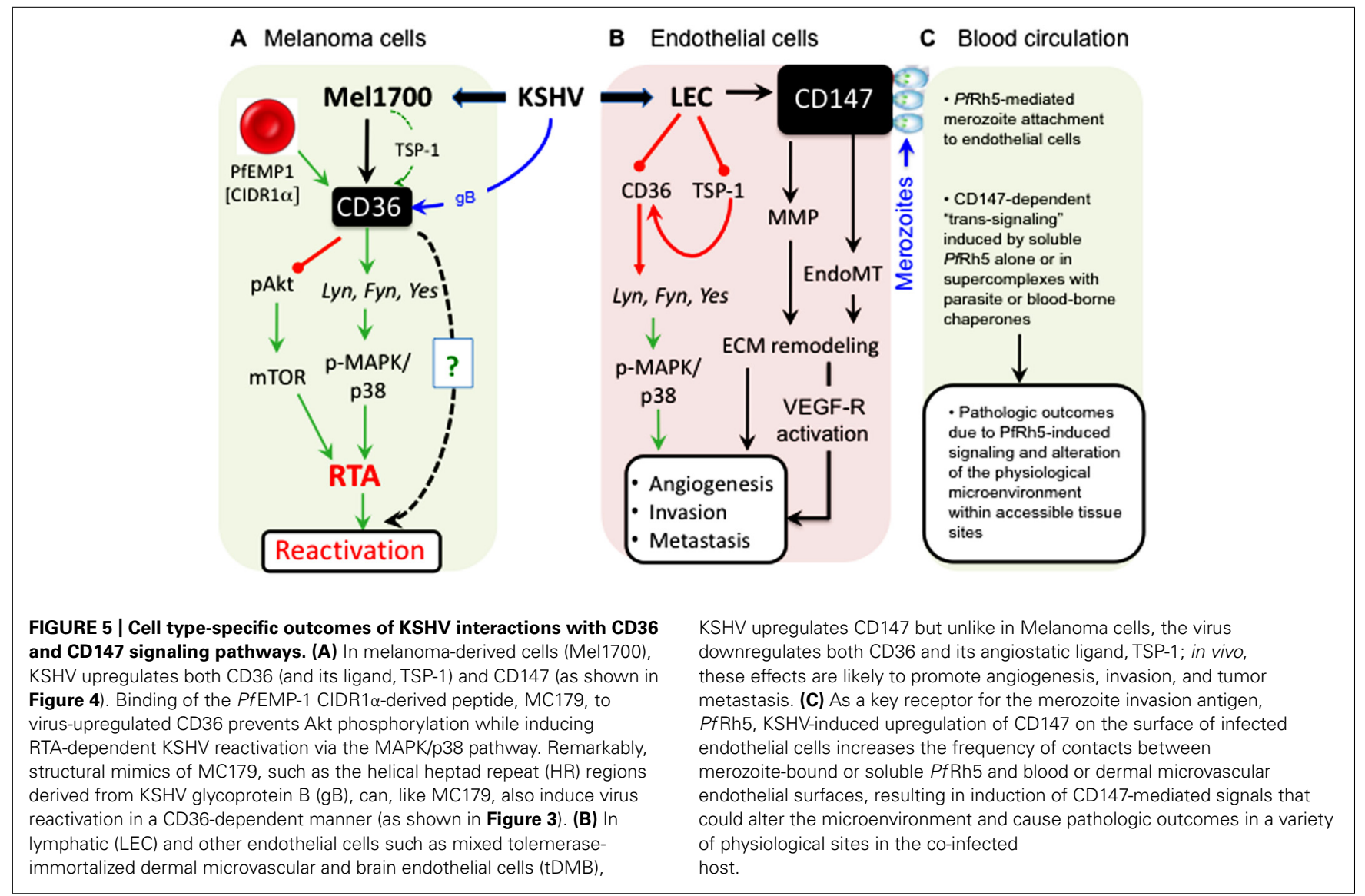




\section{THERAPEUTIC CONSIDERATIONS FOR KS AND MALARIA: CHALLENGES AND NEW OPPORTUNITIES}

Kaposi's sarcoma is one of the most frequent neoplasia diagnosed in malaria-endemic regions of Africa, yet despite recent progress (Casper, 2006; Casper and Wald, 2007), the landscape of effective therapeutic strategies for KS remains limited. It is clear that KS presents in different epidemiologic and clinical forms dictated by a variety of modifying risk factors, but the lack of data on the relative contributions of these co-factors in any given epidemiological setting frustrates efforts aimed at developing new approaches for clinical management of the disease. Surgical removal of isolated nodular KS does not eliminate latent KSHV at secondary sites, while traditional chemotherapy is generally toxic and may have a high failure rate in $\mathrm{HIV}$-infected patients in whom durable post-therapy immune reconstitution is improbable. For epidemic (HIV/AIDS-associated) KS, intervention with the highly active anti-retroviral therapy (HAART) in HIV/HHV8 (human herpes virus 8) co-infected patients may have contributed to regression of KS lesions, but the initial success of HAART-based therapy for KS has been eroded by several concerns. First, HIV is not necessary for endemic African or classical Mediterranean KS. Second, HAART only limits the potentiating immunosuppressive effects of HIV but it does not remove the underlying etiology of KS. Third, HAART may reduce HIV viral load but it does not restore the entire $\mathrm{T}$ cell repertoire necessary for immunity against KSHV. Fourth, lack of access to HAART, non-compliance with the treatment, failure to respond to treatment, and the development of drug-resistant strains of HIV confound the overall benefit of a strictly HAARTbased approach to KS management. Fifth, the benefits of HAART are not long-lasting and end up being more detrimental to many patients with advanced KS who may show no improvement while remaining in danger of developing post-therapy immune crisis (Krown et al., 2004). Sixth, the probability of recrudescence of KS in patients treated with HAART later in life is unpredictable. There is, therefore, an urgent need for a multi-pronged therapeutic approach aimed at developing strategies that are appropriate to the prevailing epidemiologic state of the disease, with the overall goal being improvement of the treatment outcome for KS patients in sub-Saharan Africa and other resource-limited parts of the world (McAllister et al., 2005).

The concept of "angio-therapy" designed to inhibit growth of angio-proliferative cancers like KS (Tosetti et al., 2002a,b; Ferrari et al., 2003; Pfeffer etal., 2003) led to the surprising observation that the anti-malarial peptide artesunate has anti-angiogenic effects on KS-derived endothelial cell lines (Dell'Eva et al., 2004). Artesunate is already well tolerated as an anti-malarial drug, and because it has direct effects against transformed cells, the promise of its dual use for $\mathrm{KS}$ is attractive for co-infected individuals. If adopted as such, the clinical benefits of artesunate for treatment of KS would represent a classic illustration of one of the defining attributes of a syndemic relationship in which the overall clinical impact of two linked infections (i.e., $P f$ and KSHV in this case) can be blunted by targeting the molecular underpinnings that link the parasite with the disease-modifying influence of KSHV. In addition, it has been proposed that quinine and its chloroquine and hydroxychloroquine derivatives - drugs that have been used widely to treat malaria - are immunosuppressive and may, as such, serve as co-factors for KS by stimulating KSHV reactivation, which would not only promote virus dissemination but could also support KS histogenesis (Ruocco et al., 2011). However, the "oncodrug" hypothesis for KS is likely to be more relevant for individuals with severely altered immunity since, in immunocompetent hosts, the viremic state induced by quinine and its derivative drugs would concomitantly expose hematogenously disseminating virions to immune surveillance which could in turn limit virus spread.

With respect to malaria control, approaches that interrupt the parasite life cycle are ideal, yet in spite of many multi-national efforts in this regard, successful elimination of the disease remains a major challenge, as more than half of the world's population still lives in areas where there is a risk of contracting the disease. There are many reasons for this sobering report card, chief among them being persistent endemicity as a result of drug and insecticide resistance, inadequate support for malaria control programs, poor environmental management, the complex biology of the disease, as well as the regional variability not only in the parasite but also in the nature of its impact on specific populations and age groups. Another major challenge remains the lack of practical and affordable animal models that can faithfully reflect mechanisms of malaria pathogenesis and immunity in humans. Such platforms would be valuable for evaluating the efficacy of drug and vaccine candidates, and for predicting the benefits of drug combinations that can maximize safety and efficacy while minimizing the development of drug resistance.

Given that the invasive asexual blood stage of the Pf lifecycle is the form associated with symptomatic malaria, recent efforts toward a malaria vaccine have primarily focused on targeting this stage. In this respect, the discovery of the essential merozoite invasion receptor increases the number of potential targets for a second-generation malaria-specific vaccine based on CD147/PfRh5 interactions. However, the fact that deletion of other PfRh proteins also impairs invasion, albeit in a strainspecific manner, complicates vaccine design efforts, as it suggests that the PfRh5/CD147 interaction may be only one of many ligand-receptor recognition events that must occur during execution of the invasion process (Cowman and Crabb, 2006; Tanne, 2011; Tham et al., 2012). An approach that targets CD147 may not be feasible, as it could impact many important physiological processes controlled by this molecule. On the other hand, innovations oriented toward development of vaccine and therapeutic strategies based on the invariant aspects of PfRh5 and other "accessible" Pf antigens may result in a more meaningful outcome associated with minimal impact on the human host. Such strategies may include the combined use of nanovehicledeliverable peptide mimetics and single-chain antibodies that can be administered before or during active parasitemia, or therapeutic lentiviral vectors carrying immunogenic epitopes that can harness the host's immune capacity. These pathogen-centered approaches could be used alone or in conjunction with the RTS,S vaccine that is based on the most prominent surface antigen of the pre-liver sporozoite stage and which has already shown some promise in phase III trials (Tanne, 2011). In using PfRh5 as the target, however, the primary goal of any given approach [antibody-based (Douglas et al., 2011), or otherwise] would be 
to elicit the safest, most long-lasting and most efficacious outcome that limits the availability of PfRh5 to mediate invasion, but it must also be guided by the recognition that this antigen is predominantly located within the rhoptries and it is liberated and or revealed to the immune system only for a short period of time when the merozoite contacts the erythrocyte prior to invasion.

\section{CONCLUSIONS AND FUTURE PERSPECTIVES}

Infectious agents have long been implicated in the etiology of a variety of illnesses, and although recent studies have examined the role of microbial co-infections in many disease settings, it is not known whether "cooperative pathogenesis" is sufficient to provide the driving force behind strategies in which co-pathogenic agents co-evolve and forge a state of forbearance with each other and with their shared host to advance mutually exclusive existential goals. Clearly, $P f$ malaria has been linked to a variety of other infections that display co-pathogenic relationships with the parasite, notable among them being eBL (Thorley-Lawson and Allday, 2008), but recent advances have revealed that many other important examples of these relationships do exist, and in most cases they provide a surprisingly informative conceptual window into how co-infections can modify each other's disease course. For instance, the recent elucidation of the molecular mechanisms of erythrocyte invasion and cyto-adherence has: (a) illustrated the extent to which $P f$ can exert its impact on human physiology; (b) exposed how alterations in the expression and function of the host receptors that support these processes could not only dramatically change the dynamics of malaria but may also influence the pathogenesis of other coinfections such as KSHV that exploit these pathways for existential benefit; and (c) generated new interest in the structural dispositions of these receptor/ligand pairs as potential multi-domain vaccine targets against specific stages of the Pflife cycle.
Although malaria is not considered a typical opportunistic infection in the same way that KSHV is, recent advances have revealed a surprising node of intersection between KSHV and malaria pathogenesis at the level of the molecular controls that regulate the persistence of these two highly successful infectious agents. To the extent that such interactions can be measured at a micro level, the concept of co-pathogenesis establishes grounds for the expectation that KSHV and $P f$ can bidirectionally influence the clinical course of each other at many physiological levels leading to a variety of clinical outcomes (Table 1; Figure 5). For example, CD36-mediated cyto-adherence, by virtue of its ability to stimulate pathways that overlap with those required for KSHV reactivation, may contribute to a transient increase in KSHV viral load and dissemination, which in turn could increase the frequency of other defining correlates of KSHV-associated disease that rely on a viremic state. On the other hand, the pathogenic mechanisms of a latent $\mathrm{KSHV}$ infection, which include KSHV-induced downregulation of CD36, could effectively alter the overall disease burden by limiting peripheral sequestration; this could in turn increase parasitic access to the central nervous system, leading to a higher probability for cerebral malaria. Ultimately, the emerging picture supports a syndemic link which, however serendipitous, reveals a co-evolutionary paradigm centered at the putative dueling role of CD36 as a mediator of parasite sequestration on one hand and KSHV replication on the other. In this regard, more extensive molecular and genetic analysis is required in order to determine the extent to which KSHV (or EBV, for that matter) might provide the driving force for altering the genetic registry at the CD36 locus in regions of high malaria endemicity. It might also be necessary to analyze the CD36 locus in hematopoietic versus peripheral B cells that may serve as the vehicle for virus dissemination in vivo, in order to determine whether infection increases the propensity for a heritable lesion at this locus.

Table 1 | Summary of the effects of KSHV on CD36 and CD147 expression and signaling, and examples of their potential impact on KSHV and $P$ falciparum disease pathogenesis.

\begin{tabular}{|c|c|c|c|c|}
\hline Cell type & $\begin{array}{l}\text { Target } \\
\text { molecule }\end{array}$ & $\begin{array}{l}\text { Effect of } \\
\text { KSHV }\end{array}$ & Potential impact on KSHV pathogenesis & Potential impact on $P$ falciparum \\
\hline LEC) & $\begin{array}{l}\text { CD36 } \\
\text { TSP-1 }\end{array}$ & $\begin{array}{l}\text { Down } \\
\text { Down }\end{array}$ & $\begin{array}{l}\text { - Associated with establishment of latency } \\
\text { - Promotes the angiogenic phenotype }\end{array}$ & $\begin{array}{l}\text { - Reduced peripheral sequestration } \\
\text { - Enhanced probability for cerebral malaria }\end{array}$ \\
\hline $\begin{array}{l}\text { Skin-derived } \\
\text { (Melanoma) }\end{array}$ & CD147 & Up & - Indeterminate & $\begin{array}{l}\text { - Increase in merozoite invasion and para- } \\
\text { sitemia }\end{array}$ \\
\hline $\begin{array}{l}\text { cells (Mel1700 } \\
\text { and Sk-Mel28) }\end{array}$ & CD36 & Up & $\begin{array}{l}\text { - The increase in binding of PfEMP-1 to upregulated CD36 } \\
\text { leads to virus reactivation }\end{array}$ & $\begin{array}{l}\text { - Increase in CD36/PfEMP-1 binding may } \\
\text { lead to enhanced sequestration of } \\
\text { parasitized erythrocytes }\end{array}$ \\
\hline
\end{tabular}

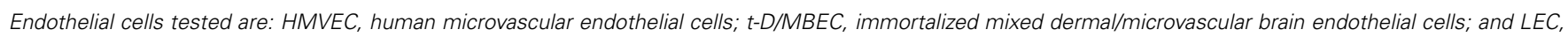
Lymphatic endothelial cells. Skin (melanoma)-derived cells tested are Mel1700 and Sk-Mel28 (ATCC). TSP-1, Thrombospondin-1. 
In conclusion, recent advances have revealed several nodes of pathobiological intersection between malaria and a variety of clinically significant infections, and although substantial progress has been made, we are still in the "embryonic stage" of understanding how co-infections interact with each other in their mutual host. Derivative new research emphasis that is inspired by these concepts should include the important goal of developing practical in vivo platforms (animal models) that could facilitate systematic, experimental integration of population studies with reductionist multi-component molecular data. Although we are still a long way from developing such a platform for studying the malaria and KSHV co-infection paradigm, attempts toward this goal are an essential step in elucidating the extent to which triangular interactions between $P f$, KSHV, and their mutual human host might articulate a syndemic relationship that underlies the

\section{REFERENCES}

Aitman, T. J., Cooper, L. D., Norsworthy, P. J., Wahid, F. N., Gray, J. K., Curtis, B. R., et al. (2000). Malaria susceptibility and CD36 mutation. Nature 405, 1015-1016.

Akula, S. M., Pramod, N. P., Wang, F. Z., and Chandran, B. (2002). Integrin alpha3betal (CD 49c/29) is a cellular receptor for Kaposi's sarcomaassociated herpesvirus (KSHV/HHV8 ) entry into the target cells. Cell 108, 407-419.

Ascoli, V., Belli, S., Benedetti, M., Trinca, S., Ricci, P., and Comba, P. (2001). High incidence of classic Kaposi's sarcoma in Mantua, Po Valley, Northern Italy (1989-1998). Br. J. Cancer 85, 379-382.

Ascoli, V., Facchinelli, L., Valerio, L., Manno, D., and Coluzzi, M. (2006a). Kaposi's sarcoma, human herpesvirus 8 infection and the potential role of promoterarthropod bites in northern Sweden. J. Med. Virol. 78, 14521455.

Ascoli, V., Facchinelli, L., Valerio, L., Zucchetto, A., Dal Maso, L., and Coluzzi, M. (2006b). Distribution of mosquito species in areas with high and low incidence of classic Kaposi's sarcoma and seroprevalence for HHV-8. Med. Vet. Entomol. 20, 198-208.

Ascoli, V., Manno, D., and Coluzzi, M. (2006c). Geographic variation in human herpesvirus 8 seroprevalence and possible association with exposure to bites from blood-sucking arthropods. J. Infect. Dis. 194, 401402; author reply 402.

Bagavandoss, P., and Wilks, J. W. (1990). Specific inhibition of endothelial cell proliferation by thrombospondin. Biochem. Biophys. Res. Commun. 170, 867-872.

Baruch, D. I., Ma, X. C., Singh, H. B., Bi, X., Pasloske, B. L., and Howard, R.
J. (1997). Identification of a region of PfEMP1 that mediates adherence of Plasmodium falciparum infected erythrocytes to CD36: conserved function with variant sequence. Blood 90 , 3766-3775.

Baruch, D. I., Pasloske, B. L., Singh, H. B., Bi, X., Ma, X. C., Feldman, M., et al. (1995). Cloning the P. falciparum gene encoding PfEMP1, a malarial variant antigen and adherence receptor on the surface of parasitized human erythrocytes. Cell 82, 77-87.

Basilico, N., Mondani, M., Parapini, S., Speciale, L., Ferrante, P., and Taramelli, D. (2004). Plasmodium falciparum parasitized red blood cells modulate the production of endothelin-1 by human endothelial cells. Minerva Med. 95, 153-158.

Bassett, M. T., Levy, L., Chokunonga, E., D. M. (1995). Cancer in the European population of Harare, Zimbabwe, 1990-1992. Int. J. Cancer 63, 24-28.

Baum, J., Chen, L., Healer, J., Lopaticki, S., Boyle, M., Triglia, T., et al. (2009). Reticulocyte-binding protein homologue 5 - an essential adhesin involved in invasion of human erythrocytes by Plasmodium falciparum. Int. J. Parasitol. 39, 371-380.

Berendt, A. R. (1993). Sequestration and its discontents: infected actions in Plasmodium falciparum malaria. Res. Immunol. 144, 740-745; discussion 754-762.

Biswas, C., Zhang, Y., DeCastro, R., Guo, H., Nakamura, T., Kataoka, H., et al. (1995). The human tumor cell-derived collagenase stimulatory factor (renamed EMMPRIN) is a member of the immunoglobulin superfamily. Cancer Res. 55, 434-439.

Bougatef, F., Quemener, C., Kellouche, S., Naimi, B., Podgorniak, M. P., Mauchaza, B., Ferlay, J., and Parkin, erythrocyte-endothelial cell inter-

co-incidence of aggressive KS in parts of the world with endemic malaria. Once the correlates of co-pathogenesis are isolated, innovative research efforts oriented toward development of effective "combined" therapies can be launched.

\section{ACKNOWLEDGMENTS}

The authors wish to express special thanks to Dr. Louis Miller, Dr. David Narum, and Dr. Morris Makobongo for expert advice and for providing recombinant MC179 proteins, and to Anita Marinelli and other members of the Johnan A. R. Kaleeba laboratory for technical assistance and constructive discussions. Work in the authors' laboratory is supported by a grant from the U.S. Department of Defense (R073NS and R073RZ to Johnan A. R. Kaleeba) through the Intramural Award Program of the Uniformed Services University of the Health Sciences.

Millot, G., et al. (2009). EMMPRIN promotes angiogenesis through hypoxia-inducible factor-2alphamediated regulation of soluble VEGF isoforms and their receptor VEGFR-2. Blood 114, 55475556.

Bubman, D., and Cesarman, E. (2003). Pathogenesis of Kaposi's sarcoma. Hematol. Oncol. Clin. North Am. 17, 717-745.

Casper, C. (2006). Defining a role for antiviral drugs in the treatment of persons with HHV-8 infection. Herpes $13,42-47$

Casper, C., and Wald, A. (2007). The use of antiviral drugs in the prevention and treatment of Kaposi sarcoma, multicentric Castleman disease and primary effusion lymphoma. Curr. Top. Microbiol. Immunol. 312 289-307.

Chen, L., Lopaticki, S., Riglar, D. T., Dekiwadia, C., Uboldi, A. D. Tham, W. H., et al. (2011). An EGFlike protein forms a complex with $\mathrm{PfRh} 5$ and is required for invasion of human erythrocytes by Plasmodium falciparum. PLoS Pathog. 7:e1002199. doi: 10.1371/journal.ppat.1002199

Chen, Q., Heddini, A., Barragan, A., Fernandez, V., Pearce, S. F., and Wahlgren, M. (2000). The semiconserved head structure of Plasmodium falciparum erythrocyte membrane protein 1 mediates binding to multiple independent host receptors. J. Exp. Med. 192, 1-10.

Chen, Z., Mi, L., Xu, J., Yu, J., Wang, X., Jiang, J., et al. (2005). Function of HAb18G/CD147 in invasion of host cells by severe acute respiratory syndrome coronavirus. J. Infect. Dis. 191, 755-760.

Chene, A., Donati, D., GuerreiroCacais, A. O., Levitsky, V., Chen, Q., Falk, K. I., etal. (2007). A molecular link between malaria and Epstein-Barr virus reactivation. PLoS
Pathog. 3:e80. doi: 10.1371/journal.ppat.0030080

Cheng, F., Pekkonen, P., Laurinavicius, S., Sugiyama, N., Henderson, S., Gunther, T., et al. (2011). KSHV-initiated notch activation leads to membrane-type-1 matrix metalloproteinase-dependent lymphatic endothelial-to-mesenchymal transition. Cell Host Microbe 10, 577-590.

Chilongola, J., Balthazary, S., Mpina, M., Mhando, M., and Mbugi, E. (2009). CD36 deficiency protects against malarial anaemia in children by reducing Plasmodium falciparuminfected red blood cell adherence to vascular endothelium. Trop. Med. Int. Health 14, 810-816.

Cohen, A., Brodie, C., and Sarid, R. (2006). An essential role of ERK signalling in TPA-induced reactivation of Kaposi's sarcoma-associated herpesvirus. J. Gen. Virol. 87, 795-802.

Coluzzi, M., Calabro, M. L., Manno, D., Chieco-Bianchi, L., Schulz, T. F., and Ascoli, V. (2003). Reduced seroprevalence of Kaposi's sarcoma-associated herpesvirus (KSHV), human herpesvirus 8 (HHV8), related to suppression of Anopheles density in Italy. Med. Vet. Entomol. 17, 461-464.

Coluzzi, M., Calabro, M. L., Manno, D., Chieco-Bianchi, L., Schulz, T. F., and Ascoli, V. (2004a). HHV8 transmission via saliva to soothe blood-sucking arthropod bites. $\mathrm{Br}$. J. Cancer 91, 998-999; author reply 999. Coluzzi, M., Calabro, M. L., Manno, D., Chieco-Bianchi, L., Schulz, T. F., and Ascoli, V. (2004b). Saliva and the transmission of human herpesvirus 8: potential role of promoter-arthropod bites. J. Infect. Dis. 190, 199-200; author reply 200-201.

Cowman, A. F., and Crabb, B. S. (2006). Invasion of red blood cells by malaria parasites. Cell 124, 755-766. 
Crombie, R., Kawasaki, K., Hojo, K., and Laurence, J. (2001). Peptides derived from salivary thrombospondin-1 replicate its anti-HIV effect: potential role in microbicide development. J. Acquir. Immune Defic. Syndr. 27, 91-93.

Crombie, R., Silverstein, R. L., MacLow, C., Pearce, S. F., Nachman, R. L., and Laurence, J. (1998). Identification of a CD36-related thrombospondin 1-binding domain in HIV-1 envelope glycoprotein gp120: relationship to HIV-1-specific inhibitory factors in human saliva. J. Exp. Med. 187, 25-35.

Crosnier, C., Bustamante, L. Y., Bartholdson, S. J., Bei, A. K., Theron, M., Uchikawa, M., etal. (2011). Basigin is a receptor essential for erythrocyte invasion by Plasmodium falciparum. Nature 480, 534-537.

Dai, L., Bratoeva, M., Toole, B. P., Qin, Z., and Parsons, C. (2012a). KSHV activation of VEGF secretion and invasion for endothelial cells is mediated through viral upregulation of emmprin-induced signal transduction. Int. J. Cancer 131, 834-843.

Dai, L., Qin, Z., Defee, M., Toole, B. P., Kirkwood, K. L., and Parsons, C. (2012b). Kaposi sarcoma-associated herpesvirus (KSHV) induces a functional tumor-associated phenotype for oral fibroblasts. Cancer Lett. 318, 214-220.

Dell'Eva, R., Pfeffer, U., Vene, R., Anfosso, L., Forlani, A., Albini, A. et al. (2004). Inhibition of angiogenesis in vivo and growth of Kaposi's sarcoma xenograft tumors by the anti-malarial artesunate. Biochem. Pharmacol. 68, 2359-2366.

Donati, D., Zhang, L. P., Chene, A., Chen, Q., Flick, K., Nystrom, M., et al. (2004). Identification of a polyclonal B-cell activator in Plasmodium falciparum. Infect. Immun. 72, 5412-5418.

Douglas, A. D., Williams, A. R., Illingworth, J. J., Kamuyu, G., Biswas, S., Goodman, A. L., et al. (2011). The blood-stage malaria antigen PfRH5 is susceptible to vaccine-inducible cross-strain neutralizing antibody. Nat. Commun. 2, 601.

Dourmishev, L. A., Dourmishev, A. L., Palmeri, D., Schwartz, R. A., and Lukac, D. M. (2003). Molecular genetics of Kaposi's sarcomaassociated herpesvirus (human herpesvirus-8) epidemiology and pathogenesis. Microbiol. Mol. Biol. Rev. 67, 175-212.

Ferrari, N., Morini, M., Pfeffer, U., Minghelli, S., Noonan, D. M., and Albini, A. (2003). Inhibition of Kaposi's sarcoma in vivo by fenretinide. Clin. Cancer Res. 9, 60206029.

Fried, M., and Duffy, P. E. (1998). Maternal malaria and parasite adhesion. J. Mol. Med. (Berl.) 76, 162-171.

Fry, A. E., Ghansa, A., Small, K. S., Palma, A., Auburn, S., Diakite, M., et al. (2009). Positive selection of a CD36 nonsense variant in sub-Saharan Africa, but no association with severe malaria phenotypes. Hum. Mol. Genet. 18, 2683 2692.

Gallagher, S. M., Castorino, J. J., and Philp, N. J. (2009). Interaction of monocarboxylate transporter 4 with betal-integrin and its role in cell migration. Am. J. Physiol. Cell Physiol. 296, C414-C421.

Ganem, D. (2006). KSHV infection and the pathogenesis of Kaposi's sarcoma. Annu. Rev. Pathol. 1, 273-296.

Gao, X., Ikuta, K., Tajima, M. and Sairenji, T. (2001). 12-Otetradecanoylphorbol-13-acetate induces Epstein-Barr virus reactivation via NF-kappaB and $\mathrm{AP}-1$ as regulated by protein kinase $\mathrm{C}$ and mitogen-activated protein kinase. Virology 286, 91-99.

Gasperini, P., Espigol-Frigole, G., McCormick, P. J., Salvucci, O., Maric, D., Uldrick, T. S., et al. (2012). Kaposi sarcoma herpesvirus promotes endothelial-to-mesenchymal transition through Notch-dependent signaling. Cancer Res. 72, 1157-1169.

Gelhaus, A., Scheding, A., Browne, E., Burchard, G. D., and Horstmann, R. D. (2001). Variability of the CD36 gene in West Africa. Hum. Mutat. 18, 444-450.

Goedert, J. J., Vitale, F., Lauria, C., Serraino, D., Tamburini, M., Montella, M., et al. (2002). Risk factors for classical Kaposi's sarcoma. J. Natl. Cancer Inst. 94, 1712-1718.

Good, D. J., Polverini, P. J., Rastinejad, F., Le Beau, M. M., Lemons, R. S. Frazier, W. A., et al. (1990). A tumor suppressor-dependent inhibitor of angiogenesis is immunologically and functionally indistinguishable from a fragment of thrombospondin. Proc. Natl. Acad. Sci. U.S.A. 87, 66246628.

Grundhoff, A., and Ganem, D. (2004). Inefficient establishment of KSHV latency suggests an additional role for continued lytic replication in Kaposi sarcoma pathogenesis. J. Clin. Invest. 113, 124-136.

Guo, H., Li, R., Zucker, S., and Toole, B. P. (2000). EMMPRIN (CD147), an inducer of matrix metalloproteinase synthesis, also binds interstitial collagenase to the tumor cell surface. Cancer Res. 60, 888-891.
Hadinoto, V., Shapiro, M., Sun, C. C., and Thorley-Lawson, D. A. (2009). The dynamics of EBV shedding implicate a central role for epithelial cells in amplifying viral output. PLoS Pathog. 5:e1000496. doi: 10.1371/journal.ppat.1000496

Haverkos, H. W. (2008). Multifactorial etiology of Kaposi' sarcoma: a hypothesis. J. Biosci. 33, 643-651.

Hengge, U. R., Ruzicka, T., Tyring, S. K., Stuschke, M., Roggendorf, M., Schwartz, R. A., et al. (2002a). Update on Kaposi's sarcoma and other HHV8 associated diseases. Part 1: epidemiology, environmental predispositions, clinical manifestations, and therapy. Lancet Infect. Dis. 2, 281-292.

Hengge, U. R., Ruzicka, T., Tyring, S. K., Stuschke, M., Roggendorf, M. Schwartz, R. A., et al. (2002b). Update on Kaposi's sarcoma and other HHV8 associated diseases. Part 2: pathogenesis, Castleman's disease, and pleural effusion lymphoma. Lancet Infect. Dis. 2, 344-352.

Horenstein, M. G., Nador, R. G., Chadburn, A., Hyjek, E. M., Inghirami, G., Knowles, D. M. et al. (1997). Epstein-Barr virus latent gene expression in primary effusion lymphomas containing Kaposi's sarcoma-associated herpesvirus/human herpesvirus-8. Blood 90, 1186-1191.

Huang, Y., Dai, Z., Barbacioru, C. and Sadee, W. (2005). Cystineglutamate transporter SLC7A11 in cancer chemosensitivity and chemoresistance. Cancer Res. 65, 7446-7454.

Iruela-Arispe, M. L., Bornstein, P., and Sage, H. (1991). Thrombospondin exerts an antiangiogenic effect on cord formation by endothelial cells in vitro. Proc. Natl. Acad. Sci. U.S.A. 88, 5026-5030.

Kaleeba, J. A., and Berger, E. A. (2006). Kaposi's sarcoma-associated herpesvirus fusion-entry receptor: cystine transporter xCT. Science 311, 1921-1924.

Kanekura, T., and Chen, X. (2010). CD147/basigin promotes progression of malignant melanoma and other cancers. J. Dermatol. Sci. 57, 149-154.

Kestens, L., Melbye, M., Biggar, R. J., Stevens, W. J., Piot, P., De Muynck, A., et al. (1985). Endemic African Kaposi's sarcoma is not associated with immunodeficiency. Int. J. Cancer 36, 49-54.

Krown, S. E., Northfelt, D. W., Osoba, D., and Stewart, J. S. (2004). Use of liposomal anthracyclines in Kaposi's sarcoma. Semin. Oncol. 31, 36-52.
Lin, C. J., Katongole-Mbidde, E., Byekwaso, T., Orem, J., Rabkin, C. S., and Mbulaiteye, S. M. (2008). Intestinal parasites in Kaposi sarcoma patients in Uganda: indication of shared risk factors or etiologic association. Am. J. Trop. Med. Hyg. 78, 409-412.

Lopaticki, S., Maier, A. G., Thompson, J., Wilson, D. W., Tham, W. H., Triglia, T., et al. (2011). Reticulocyte and erythrocyte binding-like proteins function cooperatively in invasion of human erythrocytes by malaria parasites. Infect. Immun. 79, 11071117.

Luka, J., Kallin, B., and Klein, G. (1979). Induction of the Epstein-Barr virus (EBV) cycle in latently infected cells by $n$-butyrate. Virology 94 , 228-231.

Marieb, E. A., Zoltan-Jones, A., Li, R., Misra, S., Ghatak, S., Cao, J., etal. (2004). Emmprin promotes anchorage-independent growth in human mammary carcinoma cells by stimulating hyaluronan production. Cancer Res. 64, 1229-1232.

McAllister, S. C., Fruh, K., and Moses, A. V. (2005). Functional genomics and the development of pathogenesistargeted therapies for Kaposi's sarcoma. Pharmacogenomics 6, 235-244.

McGilvray, I. D., Serghides, L., Kapus, A., Rotstein, O. D., and Kain, K. C. (2000). Nonopsonic monocyte/macrophage phagocytosis of Plasmodium falciparum-parasitized erythrocytes: a role for CD36 in malarial clearance. Blood 96, 32313240 .

Montella, M., Franceschi, S., Geddes, M., Arniani, S., and Cocchiarella, G. (1996). Classic Kaposi's sarcoma and volcanic soil in southern Italy. Lancet 347, 905.

Montella, M., Franceschi, S., Geddes da Filicaia, M., De Macro, M., Arniani, S., Balzi, D., et al. (1997). Classical Kaposi sarcoma and volcanic soil in southern Italy: a case-control study. Epidemiol. Prev. 21, 114-117.

Montella, M., Serraino, D., Crispo, A., Rezza, G., Carbone, S., and Tamburini, M. (2000). Is volcanic soil a cofactor for classic Kaposi's sarcoma? Eur. J. Epidemiol. 16, 1185-1186.

Montesu, M. A., De Marco, R., and Cottoni, F. (1995). Soil silicates and Kaposi's sarcoma in Sardinia. Lancet 346, 1436-1437.

Moormann, A. M., Snider, C. J., and Chelimo, K. (2011). The company malaria keeps: how co-infection with Epstein-Barr virus leads to endemic Burkitt lymphoma. Curr. Opin. Infect. Dis. 24, 435-441.

Nelson, J., Bagnato, A., Battistini, B., and Nisen, P. (2003). The endothelin axis: 
emerging role in cancer. Nat. Rev. Cancer 3, 110-116.

Ockenhouse, C. F., Ho, M., Tandon, N. N., Van Seventer, G. A., Shaw, S., White, N. J., et al. (1991). Molecular basis of sequestration in severe and uncomplicated Plasmodium falciparum malaria: differential adhesion of infected erythrocytes to CD36 and ICAM-1. J. Infect. Dis. 164, 163-169.

Ockenhouse, C. F., Tandon, N. N., Magowan, C., Jamieson, G. A., and Chulay, J. D. (1989). Identification of a platelet membrane glycoprotein as a falciparum malaria sequestration receptor. Science 243, 1469-1471.

Okuno, S., Sato, H., KuriyamaMatsumura, K., Tamba, M., Wang, H., Sohda, S., et al. (2003). Role of cystine transport in intracellular glutathione level and cisplatin resistance in human ovarian cancer cell lines. $\mathrm{Br}$. J. Cancer 88, 951-956.

Pauk, J., Huang, M. L., Brodie, S. J., Wald, A., Koelle, D. M., Schacker, T., et al. (2000). Mucosal shedding of human herpesvirus 8 in men. N. Engl. J. Med. 343, 1369-1377.

Pfeffer, U., Ferrari, N., Morini, M., Benelli, R., Noonan, D. M., and Albini, A. (2003). Antiangiogenic activity of chemopreventive drugs. Int. J. Biol. Markers 18, 70-74.

Piriou, A. S., Asito, P. O., Sumba, N., Fiore, J. M., Middeldorp, A. M., Moormann, R., et al. (2012). Early age at time of primary Epstein-Barr virus infection results in poorly controlled viral infection in infants from Western Kenya: clues to the etiology of endemic Burkitt lymphoma. J. Infect. Dis. 205, 906-913.

Podrez, E. A., Byzova, T. V., Febbraio, M., Salomon, R. G., Ma, Y., Valiyaveettil, M., et al. (2007). Platelet CD36 links hyperlipidemia, oxidant stress and a prothrombotic phenotype. Nat. Med. 13, 1086-1095.

Pushkarsky, T., Zybarth, G., Dubrovsky, L., Yurchenko, V., Tang, H., Guo, H., et al. (2001). CD147 facilitates HIV-1 infection by interacting with virusassociated cyclophilin A. Proc. Natl. Acad. Sci. U.S.A. 98, 6360-6365.

Pyakurel, P., Pak, F., Mwakigonja, A. R., Kaaya, E., and Biberfeld, P. (2007). KSHV/HHV-8 and HIV infection in Kaposi's sarcoma development. Infect. Agent Cancer 2, 4.

Qian, L. W., Xie, J., Ye, F., and Gao, S. J. (2007). Kaposi's sarcoma-associated herpesvirus infection promotes invasion of primary human umbilical vein endothelial cells by inducing matrix metalloproteinases. J. Virol. 81, 7001-7010.
Qin, Z., Dai, L., Bratoeva, M., Slomiany, M. G., Toole, B. P., and Parsons, C. (2011). Cooperative roles for emmprin and LYVE-1 in the regulation of chemoresistance for primary effusion lymphoma. Leukemia 25, 1598 1609.

Qin, Z., Dai, L., Slomiany, M. G., Toole, B. P., and Parsons, C. (2010). Direct activation of emmprin and associated pathogenesis by an oncogenic herpesvirus. Cancer Res. 70, 3884-3889.

Renne, R., Zhong, W., Herndier, B. McGrath, M., Abbey, N., Kedes, D., etal. (1996). Lytic growth of Kaposi's sarcoma-associated herpesvirus (human herpesvirus 8 ) in culture. Nat. Med. 2, 342-346.

Riethdorf, S., Reimers, N., Assmann, V., Kornfeld, J. W., Terracciano, L. Sauter, G., et al. (2006). High incidence of EMMPRIN expression in human tumors. Int. J. Cancer 119 1800-1810.

Riglar, D. T., Richard, D., Wilson, D. W., Boyle, M. J., Dekiwadia, C., Turnbull, L., et al. (2011). Superresolution dissection of coordinated events during malaria parasite invasion of the human erythrocyte. Cell Host Microbe 9, 9-20.

Rochford, R., Cannon, M. J., and Moormann, A. M. (2005). Endemic Burkitt's lymphoma: a polymicrobial disease? Nat. Rev. Microbiol. 3, 182-187.

Rodriguez, M., Lustigman, S., Montero, E., Oksov, Y., and Lobo, C. A. (2008). PfRH5: a novel reticulocytebinding family homolog of Plasmodium falciparum that binds to the erythrocyte, and an investigation of its receptor. PLoS ONE 3:e3300. doi: 10.1371/journal.pone.0003300

Rosano, L., Spinella, F., Di Castro, V., Nicotra, M. R., Albini, A., Natali, P. G., et al. (2003). Endothelin receptor blockade inhibits molecular effectors of Kaposi's sarcoma cell invasion and tumor growth in vivo. Am. J. Pathol. 163, 753-762.

Ruiz, S., Castro-Castro, A., and Bustelo, X. R. (2008). CD147 inhibits the nuclear factor of activated T-cells by impairing Vav1 and Racl downstream signaling. J. Biol. Chem. 283 5554-5566.

Ruocco, V., Ruocco, E., Schwartz, R. A., and Janniger, C. K. (2011). Kaposi sarcoma and quinine: a potentially overlooked triggering factor in millions of Africans. J. Am. Acad. Dermatol. 64, 434-436.

Samols, M. A., Skalsky, R. L., Maldonado, A. M., Riva, A., Lopez, M. C., Baker, H. V., et al. (2007). Identification of cellular genes targeted by KSHV-encoded microRNAs. PLoS Pathog. 3:e65. doi: 10.1371/journal. ppat.0030065

Shugars, D. C. (1999). Endogenous mucosal antiviral factors of the oral cavity. J. Infect. Dis. 179(Suppl. 3), S431-S435.

Sidhu, S. S., Nawroth, R., Retz, M., Lemjabbar-Alaoui, H., Dasari, V., and Basbaum, C. (2010). EMMPRIN regulates the canonical Wnt/beta-catenin signaling pathway, a potential role in accelerating lung tumorigenesis. Oncogene 29, 41454156.

Silverstein, R. L., and Febbraio, M. (2007). CD36-TSP-HRGP interactions in the regulation of angiogenesis. Curr. Pharm. Des. 13, 35593567.

Silverstein, R. L., and Febbraio, M. (2009). CD36, a scavenger receptor involved in immunity, metabolism, angiogenesis, and behavior. Sci. Signal. 2, re3.

Singh, S., Alam, M. M., Pal-Bhowmick, I., Brzostowski, J. A., and Chitnis, C. E. (2010). Distinct external signals trigger sequential release of apical organelles during erythrocyte invasion by malaria parasites. PLoS Pathog. 6:e1000746. doi: 10.1371/journal.ppat.1000746

Slomiany, M. G., Dai, L., Tolliver, L. B., Grass, G. D., Zeng, Y., and Toole, B. P. (2009a). Inhibition of functional hyaluronan-CD44 interactions in CD133-positive primary human ovarian carcinoma cells by small hyaluronan oligosaccharides. Clin. Cancer Res. 15, 7593 7601.

Slomiany, M. G., Grass, G. D., Robertson, A. D., Yang, X. Y., Maria, B. L. Beeson, C., et al. (2009b). Hyaluronan, CD44, and emmprin regulate lactate efflux and membrane localization of monocarboxylate transporters in human breast carcinoma cells. Cancer Res. 69, 1293-1301.

Snow, R. W., Guerra, C. A., Noor, A. M., Myint, H. Y., and Hay, S. I. (2005) The global distribution of clinical episodes of Plasmodium falciparum malaria. Nature 434, 214-217.

Staudt, M. R., and Dittmer, D. P. (2007). The Rta/Orf50 transactivator proteins of the gamma-herpesviridae. Curr. Top. Microbiol. Immunol. 312, 71-100.

Tang, Y., Nakada, M. T., Kesavan, P., McCabe, F., Millar, H., Rafferty, P., et al. (2005). Extracellular matrix metalloproteinase inducer stimulates tumor angiogenesis by elevating vascular endothelial cell growth factor and matrix metalloproteinases. Cancer Res. 65, 3193-3199.
Tanne, J. H. (2011). Trial shows vaccine halves malaria episodes in children in Africa. BMJ 343, d6832.

Tham, W. H., Healer, J., and Cowman, A. F. (2012). Erythrocyte and reticulocyte binding-like proteins of Plasmodium falciparum. Trends Parasitol. 28, 23-30.

Thorley-Lawson, D. A., and Allday, M. J. (2008). The curious case of the tumour virus: 50 years of Burkitt's lymphoma. Nat. Rev. Microbiol. 6, 913-924.

Tolsma, S. S., Volpert, O. V., Good, D. J., Frazier, W. A., Polverini, P. J., and Bouck, N. (1993). Peptides derived from two separate domains of the matrix protein thrombospondin1 have anti-angiogenic activity. J. Cell Biol. 122, 497-511.

Tosetti, F., Benelli, R., and Albini, A. (2002a). The angiogenic switch in solid tumors: clinical implications. Suppl. Tumori 1, S9-S11.

Tosetti, F., Ferrari, N., De Flora, S., and Albini, A. (2002b). Angioprevention': angiogenesis is a common and key target for cancer chemopreventive agents. FASEB J. 16, 2-14.

Trossaert, M., Dieye, A., Dieye, Y., and Sarthou, J. L. (1991). Cytoadherence of Plasmodium falciparum and complications of malaria. Dakar Med. 36, 192-197.

Urban, B. C., Ferguson, D. J., Pain, A., Willcox, N., Plebanski, M., Austyn, J. M., etal. (1999). Plasmodium falciparum-infected erythrocytes modulate the maturation of dendritic cells. Nature 400, 73-77.

Veettil, M. V., Sharma-Walia, N., Sadagopan, S., Raghu, H., Sivakumar, R., Naranatt, P. P., et al. (2006). RhoA-GTPase facilitates entry of Kaposi's sarcoma-associated herpesvirus into adherent target cells in a Src-dependent manner. J. Virol. 80, 11432-11446.

Vitale, F., Briffa, D. V., Whitby, D., Maida, I., Grochowska, A., Levin, A., et al. (2001). Kaposi's sarcoma herpes virus and Kaposi's sarcoma in the elderly populations of 3 Mediterranean islands. Int. J. Cancer 91, 588-591.

Wabinga, H. R., Parkin, D. M., WabwireMangen, F., and Nambooze, S. (2000). Trends in cancer incidence in Kyadondo County, Uganda, 19601997. Br. J. Cancer 82, 15851592.

Wakeham, K., Webb, E. L., Sebina, I., Muhangi, L., Miley, W., Johnson, W. T., et al. (2011). Parasite infection is associated with Kaposi's sarcoma associated herpesvirus (KSHV) in Ugandan women. Infect. Agent Cancer 6, 15. 
Watanabe, A., Yoneda, M., Ikeda, F., Terao-Muto, Y., Sato, H., and Kai, C. (2010). CD147/EMMPRIN acts as a functional entry receptor for measles virus on epithelial cells. J. Virol. 84, 4183-4193.

Wenisch, C., Wenisch, H., Wilairatana, P., Looareesuwan, S., Vannaphan, S., Wagner, O., etal. (1996). Big endothelin in patients with complicated Plasmodium falciparum malaria. J. Infect. Dis. 173, 1281-1284.

Whitby, D., Marshall, V. A., Bagni, R. K., Miley, W. J., McCloud, T. G., HinesBoykin, R., et al. (2007). Reactivation of Kaposi's sarcoma-associated herpesvirus by natural products from Kaposi's sarcoma endemic regions. Int. J. Cancer 120, 321-328.

Wu, J., Ru, N. Y., Zhang, Y., Li, Y., Wei, D., Ren, Z., et al. (2011). HAb18G/CD147 promotes epithelial-mesenchymal transition through TGF-beta signaling and is transcriptionally regulated by Slug. Oncogene 30, 4410-4427.

Xie, J., Ajibade, A. O., Ye, F., Kuhne, K., and Gao, S. J. (2008). Reactivation of Kaposi's sarcoma-associated herpesvirus from latency requires MEK/ERK, JNK and p38 multiple mitogen-activated protein kinase pathways. Virology 371, 139-154.

$\mathrm{Xu}$, D., and Hemler, M. E. (2005). Metabolic activation-related CD147CD98 complex. Mol. Cell. Proteomics 4, 1061-1071.

$\mathrm{Xu}$, H. Y., Qian, A. R., Shang, P., Xu, J., Kong, L. M., Bian, H. J., et al. (2007). siRNA targeted against HAb18G/CD147 inhibits MMP-2 secretion, actin and FAK expression in hepatocellular carcinoma cell line via ERK1/2 pathway. Cancer Lett. 247, 336-344.

Yang, H., Zou, W., Li, Y., Chen, B. and Xin, X. (2007). Bridge linkage role played by CD98hc of anti-tumor drug resistance and cancer metastasis on cisplatin-resistant ovarian cancer cells. Cancer Biol. Ther. 6, 942-947.

Yipp, B. G., Robbins, S. M., Resek, M. E., Baruch, D. I., Looareesuwan, S., and Ho, M. (2003). Src-family kinase signaling modulates the adhesion of Plasmodium falciparum on human microvascular endothelium under flow. Blood 101, 2850-2857.
Zhu, F. X., Cusano, T., and Yuan, Y. (1999). Identification of the immediate-early transcripts of Kaposi's sarcoma-associated herpesvirus. J. Virol. 73, 5556-5567.

Ziegler, J., Newton, R., Bourboulia, D., Casabonne, D., Beral, V., Mbidde, E., etal. (2003). Risk factors for Kaposi's sarcoma: a casecontrol study of HIV-seronegative people in Uganda. Int. J. Cancer 103 233-240.

Ziegler, J. L. (1993). Endemic Kaposi's sarcoma in Africa and local volcanic soils. Lancet 342, 1348-1351.

Ziegler, J. L., Templeton, A. C., and Vogel, C. L. (1984). Kaposi's sarcoma: a comparison of classical, endemic, and epidemic forms. Semin. Oncol. 11, 47-52.

Zou, W., Yang, H., Hou, X., Zhang, W., Chen, B., and Xin X. (2007). Inhibition of CD147 gene expression via RNA interference reduces tumor cell invasion, tumorigenicity and increase chemosensitivity to paclitaxel in $\mathrm{HO}$ 8910 pm cells. Cancer Lett. 248, 211-218.
Conflict of Interest Statement: The authors declare that the research was conducted in the absence of any commercial or financial relationships that could be construed as a potential conflict of interest.

Received: 15 November 2012; accepted: 07 February 2013; published online: 12 March 2013

Citation: Conant KL and Kaleeba JAR (2013) Dangerous liaisons: molecular basis for a syndemic relationship between Kaposi's sarcoma and P. falciparum malaria. Front. Microbiol. 4:35. doi: 10.3389/fmicb.2013.00035

This article was submitted to Frontiers in Virology, a specialty of Frontiers in Microbiology.

Copyright (C) 2013 Conant and Kaleeba. This is an open-access article distributed under the terms of the Creative Commons Attribution License, which permits use, distribution and reproduction in other forums, provided the original authors and source are credited and subject to any copyright notices concerning any thirdparty graphics etc. 